\title{
191.
}

\section{NOTE ON THE EXPANSION OF THE TRUE ANOMALY.}

[From the Quarterly Mathematical Journal, vol. II. (1858), pp. 229-232.]

IF the true anomaly and the mean anomaly are respectively denoted by $u, m$, and if $e$ be the eccentricity, then as usual $u-e \sin u=m$; and if we write

$$
\lambda=\frac{1-\sqrt{ }\left(1-e^{2}\right)}{e}
$$

and take $c$ to denote the base of the hyperbolic system of logarithms, we have

$$
u=m+2 \Sigma_{1}^{\infty} A_{r} \frac{\sin r m}{r},
$$

and

$$
A_{r}=\lambda^{r} c^{-\frac{1}{2} r e\left(\lambda-\lambda^{-1}\right)}+\lambda^{-r} c^{\frac{1}{2} r e\left(\lambda-\lambda^{-1}\right)},
$$

where, after expanding the exponentials, the negative powers of $\lambda$ are to be rejected and the term independent of $\lambda$ is to be multiplied by $\frac{1}{2}$ (see Camb. Math. Journal, t. I. [1839] p. 228 and t. III. [1843] p. 165, [4]).

It is easily seen that $e^{r}$ is the lowest power of $e$ which enters into the value of $A_{r}$ and the question arises to find the numerical coefficient of the term in question; this is readily obtained from the formula; in fact considering first a term of the form

$$
\lambda^{-r} e^{s}\left(\lambda-\lambda^{-1}\right)^{8}
$$

since $\lambda$ is itself of the order $e$, when the negative powers of $\lambda$ are reiected this is at least of the order $e^{s}$ and it is consequently to be neglected if $s>$.. But if $s<r$ all the powers of $\lambda$ are negative and the term is to be rejected. The only case to be 
considered is therefore that of $s=r$, in which case there is a term containing $e^{r}$. We thus obtain from $\lambda^{-r} c^{\frac{1}{2} r e\left(\lambda-\lambda^{-1}\right)}$ the term

$$
\frac{1}{2} \frac{r^{r} e^{r}}{2^{r} \cdot 1.2 .3 \ldots r}
$$

In the next place a term of the form $\lambda^{r} e^{s}\left(\lambda-\lambda^{-1}\right)^{s}$ is at least of the order $e^{s}$ if $s>r$, or the terms to be considered are those for which $s=$ or $<r$. But in such term the only part of the order $e^{r}$ is

$$
(-)^{8} \lambda^{r-8} e^{s},
$$

or, since neglecting higher powers of $e$ we have $\lambda=\frac{1}{2} e$, this is

$$
(-)^{s} 2^{-r+8} e^{r}
$$

and the set of terms arising from

$$
\lambda^{r} c^{-\frac{1}{2} r e(\lambda-\lambda-1)}
$$

is

$$
\frac{e^{r}}{2^{r}}\left\{1+\frac{r}{1}+\frac{r^{2}}{1.2} \ldots+\frac{r^{r-1}}{1.2 \ldots(r-1)}+\frac{1}{2} \frac{r^{r}}{1.2 \ldots r}\right\}
$$

the last term being divided by 2 because arising from a term independent of $\lambda$. Hence the first term of $A_{r}$ is

$$
\frac{e^{r}}{2^{r}}\left\{1+\frac{r}{1}+\frac{r^{2}}{1.2} \ldots+\frac{r^{r}}{1.2 \ldots r}\right\}
$$

a result which it may be remarked is contained in the general formula given in Hansen's Memoir “Entwickelung des Products u.s. w.," Leipzig Trans., t. II. p. 277 (1853).

The preceding expression is

$$
=\frac{e^{r} c^{r}}{2^{r}} \frac{1}{\Gamma(r+1)} \int_{r}^{\infty} x^{r} c^{-x} d x,
$$

and to find its value when $r$ is large, we have

$$
\begin{aligned}
\int_{r}^{\infty} x^{r} c^{-x} d x & =\int_{0}^{\infty}(y+r)^{r} e^{-y-r} d y=r^{r} c^{-r} \int_{0}^{\infty}\left(1+\frac{y}{r}\right)^{r} e^{-y} d y \\
& =r^{r} c^{-r} \int_{0}^{\infty} c^{-y+r \log \left(1+\frac{y}{r}\right)} d y \\
& =r^{r} c^{-r} \int_{0}^{\infty} c^{-\frac{y^{2}}{2 r}+\frac{y^{3}}{3 r^{2}}-8 c .} d y \\
& =r^{r} c^{-r} \int_{0}^{\infty}\left(1+\frac{y^{3}}{3 r^{2}}+\ldots\right) e^{-\frac{y^{2}}{2 r}} d y \\
& =r^{r} c^{-r} \sqrt{2 r} \int_{0}^{\infty}\left(1+\frac{2 \sqrt{2}}{3 \sqrt{r}} z^{3}+\ldots\right) e^{-z^{2}} d z
\end{aligned}
$$


or neglecting all the terms except the first, this is

$$
\begin{aligned}
& =r^{r} c^{-r} \sqrt{2 r} \int_{0}^{\infty} e^{-z^{2}} d z \\
& =\sqrt{2 \pi r} r^{r} c^{-r} .
\end{aligned}
$$

Hence multiplying by $\frac{1}{2^{r}} e^{r} c^{r} \frac{1}{\Gamma^{\prime}(r+1)}$ and observing that when $r$ is large, we have, by a well-known formula,

$$
\Gamma(r+1)=\sqrt{2 \pi r} r^{r} c^{-r},
$$

we obtain finally the result that when $r$ is large the first term of $A_{r}$ is approximately

$$
=\left(\frac{e c}{2}\right)^{r} \text {. }
$$

I take the opportunity of mentioning the following somewhat singular theorem, which seems to belong to a more general theory: viz. if $u-e \sin u=m$, then we have

$$
\log (1-e \cos u)=\frac{1}{\alpha} \log (1-\alpha e \cos \phi)
$$

where

$$
\phi-\frac{1}{\alpha} \tan \phi=m,
$$

provided that the negative powers of $\alpha$ are rejected, and $\alpha$ is then put equal to unity.

To show this, we have by Lagrange's theorem, observing that

$$
\begin{aligned}
\frac{d}{d m} F(1-e \cos m)= & e \sin m F^{\prime}(1-e \cos m), \\
F(1-e \cos u)=F(1-e \cos m)+ & \frac{e^{2}}{1} \sin ^{2} m F^{\prime}(1-e \cos m) \\
& +\frac{e^{3}}{1.2} \frac{d}{d m} \sin ^{3} m F^{\prime}(1-e \cos m)+\& c .
\end{aligned}
$$

and the coefficient of $e^{r}$ in $F(1-e \cos u)$ is

$$
\begin{aligned}
\frac{(-)^{r}}{1.2 \ldots(r-1)}\left\{\frac{1}{r} F_{r} \cos ^{r} m\right. & +\frac{r-1}{1} F_{r-1} \cos ^{r-2} m \sin ^{2} m \\
& \left.-\frac{(r-1)(r-2)}{1.2}{ }_{r-2} \frac{d}{d m}\left(\cos ^{r-3} m \sin ^{3} m\right)+\& c .\right\},
\end{aligned}
$$

where $F_{r}=F^{r}(1)$. 
Hence in particular when $F x=\log x, F_{r}=(-)^{r-1} 1.2 \ldots(r-1)$ and thence the coefficient of $e^{r}$ in $\log (1-e \cos u)$ is

$$
-\left\{\frac{1}{r} \cos ^{r} m-\frac{1}{1} \cos ^{r-2} m \sin ^{2} m-\frac{1}{1.2} \frac{d}{d m}\left(\cos ^{r-3} m \sin ^{2} m\right)-\& c .\right\},
$$

continued as long as the exponent of $\cos m$ is not negative. Now in the expansion of $\frac{1}{\alpha} \log (1-\alpha e \cos \phi)$, where $\phi-\frac{1}{\alpha} \tan \phi=m$, the coefficient of $e^{r}$ is $-\frac{1}{r} \alpha^{r-1} \cos ^{r} \phi$, and this (by Lagrange's theorem) is equal to

$$
\begin{array}{r}
-\frac{1}{r} \alpha^{r-1}\left\{\cos ^{r} m-\frac{1}{1 \cdot \alpha} r \cos ^{r-1} m \sin m \tan m-\frac{1}{1.2 \cdot \alpha^{2}} \frac{d}{d m}\left(r \cos ^{r-1} m \sin m \tan ^{2} m\right)-\& c .\right\} \\
=-\left\{\frac{1}{r} \alpha^{r-1} \cos ^{r} m-\frac{1}{1} \alpha^{r-2} \cos ^{r-2} m \sin ^{2} m-\frac{1}{1.2} \alpha^{r-3} \cos ^{r-3} m \sin ^{3} m-\& c .\right\},
\end{array}
$$

where the series is continued indefinitely; but if we reject the negative powers of $\alpha$ and then put $a$ equal to unity this is precisely equal to the former expression for the coefficient of $e^{r}$, and the formula is thus shown to be true.

2, Stone Buildings, W.C., 17th Nov., 1857. 